\title{
Nanodeposition of Materials with Complex Refractive Index in Long Period Fiber Gratings
}

\author{
Ignacio Del Villar, Ignacio R. Matías, Senior Member, IEEE, Francisco J. Arregui, Member, IEEE, \\ and Miguel Achaerandio
}

\begin{abstract}
An overlay of higher refractive index than the cladding is deposited on a Long Period Fiber Grating (LPFG). This permits to improve in a great manner the sensitivity of the device to ambient and overlay refractive index changes. This causes large shifts of the attenuation bands in the transmission spectrum. To obtain a maximum sensitivity for specific refractive indices of the overlay and the ambient, an optimum overlay thickness must be selected. If the refractive index of the overlay is complex there is an additional phenomenon of vanishing of the attenuation bands in the transmission spectrum. This occurs for specific thickness values of the overlay. The problem is analyzed with a numerical method based on LP mode approximation and coupled mode theory. Experimental results are contrasted with theoretical ones.
\end{abstract}

Index Terms-Coupled mode analysis, long period gratings, optical fiber sensors, refractive index, nanodeposition

\section{INTRODUCTION}

L ong Period Fiber Gratings (LPFGs) consist of an index modulation of the refractive index of the core of a single mode fiber (SMF), with a much longer period than Fiber Bragg Gratings (FBGs). They have found many applications during the nineties in optical communications and sensors fields. In optical communications many devices have been developed, such as gain equalizers [1], band rejection filters [2], tunable filters [3] and optical switches [4]. If compared with FBGs, they are also sensitive to measurands such as strain or temperature [5]-[8], which may alter the period of the grating or the refractive of the core or cladding. Nonetheless, modes couple in a different way, which improves the characteristics of sensors in a great manner. They are highly sensitive to the surrounding media, which also includes the drawback of a dependence on temperature. Anyway, there exist techniques for avoiding this problem [7], [8], which permits multi-parameter sensing [6]. They also present low background reflections and insertion losses, and demodulation

Manuscript received December 14, 2004. This work was supported by Spanish CICYT Research Grant TIC 2003-00909, Gobierno de Navarra and FPU MECD grant.

Ignacio Del Villar, Ignacio R. Matías, Francisco J. Arregui and Miguel Achaerandio are with the Electrical and Electronic Engineering Department of the Public University of Navarra, 31006 Pamplona Spain (e-mails: ignacio.delvillar@unavarra.es, natxo@unavarra.es, parregui@unavarra.es and acha@unavarra.es). schemes are economical. All these good properties make them adequate for more purposes than strain or temperature detection. They can be used as refractometers [9], [10], or for detection of chemical substances in the ambient [11]. Furthermore, if an overlay is deposited on the cladding, it modifies the coupling of modes [12]-[14]. If the material selected for the overlay is sensitive to a specific parameter, highly sensitive and specific devices will be obtained. Henceforward, the main purpose of this work is to analyze both theoretically and experimentally this last phenomenon.

Regarding the fabrication of LPFGs, this can be obtained with several techniques, being ultraviolet (UV) irradiation the most extended one. Others are ion implantation, irradiation by femtosecond pulses in the infrared, irradiation by $\mathrm{CO}_{2}$ lasers, diffusion of dopants into the core, relaxation of mechanical stress, and electrical discharges. A good review on these techniques can be found in [15].

Typically, the periodicity of LPFGs ranges between $100 \mu \mathrm{m}$ to $1 \mathrm{~mm}$. As a result, dips are created in the transmission spectrum at wavelengths where there is a coupling between the core and copropagating cladding modes, unlike in FBGs, where there is a coupling between contrapropagating modes. Each attenuation band presents a minimum, notated as resonance wavelength. This wavelength value is in close relation with the one that satisfies the Bragg condition between the coupled modes. A much better approximation can be obtained if the influence of the self coupling coefficient of the modes is included in the formulation [16], as it will be explained in next section. The third possibility is to solve the coupled mode equations. The consideration of all coupling coefficients will permit to obtain more exact values for the resonance wavelengths, and the transmission spectrum. The drawback of this option is a higher computational effort in comparison with the other two, where no differential equation has to be solved.

Regarding the analysis of LPFGs, two different cases have been studied so far. In the first one it is assumed that the ambient refractive index is lower than the cladding [17]. As the ambient refractive index approaches that of the cladding, the sensitivity of the resonance wavelength to variations of the ambient refractive index is higher. Then, the second case occurs when the ambient refractive index exceeds that of the cladding. The core couples with radiation modes [10], [19], [19] and the dependence of the resonance wavelength on the ambient refractive index is not so accused. Instead, the 
resonance depth is more dependent on this parameter for values close to the refractive index of the cladding [9]. In both cases, the sensitivity is higher when the ambient refractive index is similar to that of the cladding.

In the previous two cases, the cladding was surrounded by a medium of infinite thickness. In this work, a third possibility is presented. A thin overlay of higher refractive index than the cladding is deposited between the cladding and the infinite surrounding media. One of the cladding modes will be guided by the overlay if it is thick enough. This causes a reorganization of the effective indices of the modes of the cladding. As a result, there are important variations of the Bragg condition, which leads to dramatic shifts of the resonance wavelengths if we work around the thickness value where the cladding mode becomes guided by the overlay.

In addition to this, there is a second issue to keep in mind. So far, only Electrostatic Self-Assembly (ESA) [13], [14] and Langmuir Blodgett [12] techniques have been applied for the deposition of overlay thickness of tens of nanometers. In both cases the attenuation bands vanish for a range of thickness values and reappear as the thickness of the overlay increases. It was proved in [20] that ESA materials can present a high imaginary part due to scattering and material losses. If the imaginary part of the material of the overlay is considered, theoretical and experimental results agree. The vanishing of the attenuation bands coincides with the guidance of one of the cladding modes in the overlay as it will be explained in section 3. The consequence of this vanishing of modes is negative in terms of wavelength shift detection, because it limits the overall shift of the attenuation bands. Nonetheless, high variations in amplitude can be exploited in the same manner as wavelength shift in sensors applications. Furthermore, the theory presented can be applied to other structures with nanodeposition on a substrate [21].

The numerical method used for determining the wavelength that satisfies the Bragg condition between the core mode and each cladding mode, and the transmittance of the LPG is based on that described in [16]. Coupled mode theory is the basis for the calculation of LP modes in a cylindrical multilayer waveguide and it is explained in next section. In section 3 the phenomenon is analyzed and corroborated with experimental results. Finally, some conclusions are given in section 4.

\section{THEORY}

Up to now coupled mode theory has proved to be a powerful tool for simulation of LPFG structures. In [17] a three layer model is presented, where the transmission spectrum can be obtained provided the ambient refractive index is lower than the cladding. Otherwise, different approximations are necessary [10], [19], [19].

The structure analyzed in this work (see Fig. 1) presents four layers. The calculation of the modes in a cylindrical multilayer waveguide becomes a difficult and computational expensive task. To avoid this problem, in [16] a theoretical model is described based on the LP mode approximation [22].
Low LP modes of arbitrary azimutal number can be calculated for structures with arbitrary azimutal or radial refractive index profile. In [16] it is also proved that the high contrast between ambient and cladding refractive indices plays no important role in the results. For the sake of simplicity, the structure simulated presents no azimutal variation of the perturbed index profile after exposure to UV radiation. In this way, there are only interactions between $\mathrm{LP}_{\mathrm{vj}}(\mathrm{v}=0)$ modes, and each mode is not treated as two independent modes, as it is the case for $v>0$ modes.

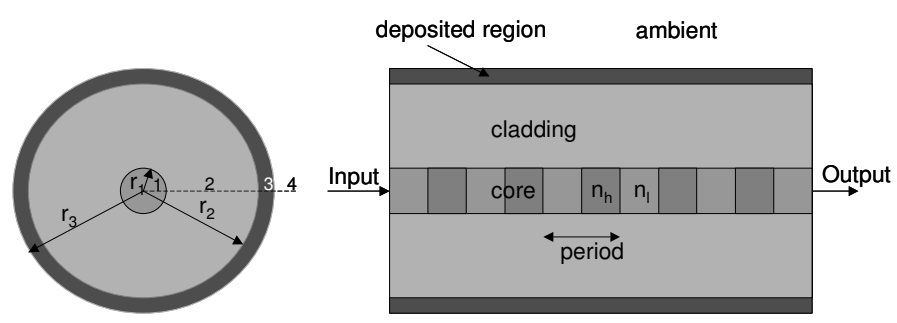

Fig. 1. Transversal and longitudinal section of LPFG structure deposition of an overlay on the cladding.

To calculate the transmission of the structure there are three main steps:

\section{A. Calculation of the propagation constants}

$\mathrm{LP}_{0 \mathrm{j}}$ modes are calculated based on the transfer matrix formulation [23]. The transverse electric field component propagating along the $\mathrm{z}$-axis is given by:

$$
\begin{aligned}
& U_{0 j, i}(r, \phi, z)=\exp \left(-j \beta_{0 j} z\right) \Psi_{0 j, i}(r, \phi)=\exp \left(-j \beta_{0 j} z\right) \Phi(\phi) R_{0 j, i}(r)= \\
& =\exp \left(-j \beta_{0 j} z\right) \times\left\{\begin{array}{c}
A_{0 j, i} J_{0}\left(r \gamma_{0 j, i}\right)+B_{0 j, i} Y_{0}\left(r \gamma_{0 j, i}\right) \\
A_{0 j, i} I_{0}\left(r \gamma_{0 j, i}\right)+B_{0 j, i} K_{0}\left(r \gamma_{0 j, i}\right)
\end{array}\right\} \text { when } \begin{array}{r}
\beta_{0 j}<k_{0} n_{i} \\
\beta_{0 j}>k_{0} n_{i}
\end{array}
\end{aligned}
$$

where $\beta_{0 \mathrm{j}}$ is the propagation constant of the $\mathrm{LP}_{0 \mathrm{j}}$ mode, $\gamma_{0 j, i}=\sqrt{\left|k_{0}^{2} n_{i}^{2}-\beta_{0 j}^{2}\right|}$ is the magnitude of the transverse wavenumber, $\phi$ is the azimutal angle, and $\mathrm{A}_{0 \mathrm{j}, \mathrm{i}}$ and $\mathrm{B}_{0 \mathrm{j}, \mathrm{i}}$ are non-normalized field expansion coefficients determined by the boundary conditions within the cylindrical layer i. $J_{0}\left(r \gamma_{0 j, i}\right)$ and $Y_{0}\left(r \gamma_{0 j, i}\right)$ are the ordinary Bessel functions of first and second kind of order 0, while $I_{0}\left(r \gamma_{0 j, i}\right)$ and $K_{0}\left(r \gamma_{0 j, i}\right)$ are the modified Bessel functions of first and second kind of order 0 .

After solving the transfer matrix method, the effective indices of the modes, and the coefficients $\mathrm{A}_{0 \mathrm{j}, \mathrm{i}}$ and $\mathrm{B}_{0 \mathrm{j}, \mathrm{i}}$ in each layer will be calculated and normalized so that each mode carries the same power $\mathrm{P}_{0}[16]$, [17]:

$P_{0 j}=\frac{\beta_{0 j}}{2 \omega \mu_{0}} \operatorname{Re} \int_{\phi=0}^{2 \pi} d \phi \int_{r=0}^{r_{1}} R_{0 j}(r) R_{0 j}^{*}(r) r d r$

\section{B. Derivation of the coupling coefficients}

According to coupled mode theory [16], [17], [24], the interaction between optical modes is proportional to their coupling coefficient. The contribution of longitudinal coupling coefficient in coupled mode analysis can be neglected [16], 
[17]. Consequently we will refer to the transversal coupling coefficient as the general coupling coefficient. In cylindrical coordinates, the coupling coefficient between each two modes can be expressed as:

$$
K_{v j, \mu k}=\frac{\omega}{4 P_{0}} \times \int_{\phi=0}^{2 \pi} \int_{r=0}^{\infty} \Delta \mathcal{E}(r, \phi, z) \Psi_{v j}(r, \phi) \Psi_{\mu k}^{*}(r, \phi) r d r d \phi
$$

where $\Psi(\mathrm{r}, \phi)$ is the transverse field for an LP mode as expressed in (1), and $\Delta \varepsilon(\mathrm{r}, \phi, \mathrm{z})$ is the permittivity variation. There is no azimutal variation of the perturbed index profile, and there is weak guidance between the core and the cladding of the fiber. Consequently, the permittivity can be expressed as:

$\Delta \varepsilon(r, z) \approx 2 \varepsilon_{0} n_{0}(r) \Delta n(r, z)$

where $\varepsilon_{0}$ is the free space permittivity, $\mathrm{n}_{0}(\mathrm{r})$ is the refractive index profile of the structure without the perturbation, and $\Delta \mathrm{n}(\mathrm{r}, \mathrm{z})$ is the variation of the refractive index. This last variable is the product of a perturbation constant and two other functions:

$\Delta n(r, z)=p(r) \sigma(z) S(z)$

where $\mathrm{p}(\mathrm{r})$ is the transverse refractive index perturbation, $\sigma(\mathrm{z})$ is the apodization factor, and $\mathrm{S}(\mathrm{z})$ is the longitudinal refractive index perturbation factor. It will be approximated by a Fourier series of two terms:

$$
S(z)=s_{0}+s_{1} \cos ((2 \pi / \Lambda) z)
$$

where $\Lambda$ is the period of the grating.

After these approximations, the coupling coefficients will be expressed as:

$K_{v j, \mu k}=\left[s_{0}+s_{1} \cos \left(\left(\frac{2 \pi}{\Lambda}\right) z\right)\right] \varsigma_{v j, \mu k}$

where

$$
\varsigma_{v j, \mu k}=\frac{\omega \varepsilon_{0}}{2 P_{0}} \times \int_{\phi=0}^{2 \pi} \int_{r=0}^{\infty} n_{0}(r) p(r) \Psi_{v j}(r, \phi) \Psi_{\mu k}^{*}(r, \phi) r d r d \phi
$$

$\varsigma_{v j, \mu k}$ is the coupling constant, even though it can show a slow ' $\mathrm{z}$ ' varying dependence [17].

Under the assumption of a uniform index perturbation within the core of the fiber, the interactions occur between $L P_{0 k}$ modes. As a result, there is no azimutal dependence and expression (8) of [16] can be simplified for the coupling coefficient:

$$
\varsigma_{0 j, 0 k}=\frac{\omega \varepsilon_{0}}{2 P_{0}} n_{0}\left(r_{1}\right) p\left(r_{1}\right) \int_{\phi=0}^{2 \pi} d \phi \int_{r=0}^{r_{1}} R_{0 j}(r) R_{0 k}^{*}(r) r d r
$$

Otherwise, the LP modes of higher azimutal order should be treated as two independent modes. An important reduction in computation time can be obtained if only half of the coupling coefficients are calculated. This can be obtained if the symmetry property is used: $\varsigma_{0 j, 0 k}=\varsigma_{0 k, 0 j}{ }^{*}$.

\section{Coupled mode equations}

After deduction of the propagation constants of the modes and the coupling coefficients, coupled mode theory will be introduced. Unlike in FBGs, backward propagating modes will be neglected. In this way, the generalized coupled mode equations describing an LPFG can be expressed as [16], [17]: $\frac{d F_{0 k}(z)}{d z}=-j \sum_{j=1}^{M} K_{v j, \mu k} F_{0 j}(z) \exp \left(-j\left(\beta_{v j}-\beta_{\mu k}\right) z\right) \quad$ for $k=1,2, \ldots M$.

This can be expressed in a matrix form in the following way:

$$
\left(\begin{array}{c}
\dot{F_{01}}(z) \\
F_{02}(z) \\
\vdots \\
\dot{F_{0 N}}(z)
\end{array}\right)=\left(\begin{array}{cccc}
Q_{01} & V_{02,01} & \cdots & V_{0 N, 01} \\
V_{01,02} & Q_{02} & \cdots & V_{0 N, 02} \\
\vdots & \vdots & \ddots & \vdots \\
V_{01,0 N} & V_{02,0 N} & \cdots & Q_{0 N}
\end{array}\right)\left(\begin{array}{c}
F_{01}(z) \\
F_{02}(z) \\
\vdots \\
F_{0 N}(z)
\end{array}\right)
$$

where $F_{0 j}$ is the normalized amplitude of the $\mathrm{j}$ mode, and the differential equation matrix elements are defined as:

$$
\begin{aligned}
& Q_{a j}=-j \sigma(z) s_{0} \zeta_{0 j, 0 j} \exp \left\lfloor-2 \cdot \operatorname{imag}\left(\beta_{0 j}\right) z\right\rfloor \\
& V_{0 j, 0 k}=-j \sigma(z) \frac{s_{1}}{2} \zeta_{0 j, 0 k} \exp \left[-j z\left(\operatorname{real}\left(\beta_{0 j}-\beta_{0 k}\right) \pm \frac{2 \pi}{\Lambda}\right)\right] \exp \left[-\operatorname{imag}\left(\beta_{0 j}+\beta_{0 k}\right)\right]
\end{aligned}
$$

where $s_{0}$ and $s_{1}$ are the coefficients of the first two Fourier components of the grating function $\mathrm{S}(\mathrm{z}), \beta_{0 \mathrm{j}}$ is the propagation constant of the $\mathrm{j}$ mode, and $\Lambda$ is the period of the grating.

The \pm sign in the first exponential functions of $\mathrm{V}_{0 \mathrm{j}, 0 \mathrm{k}}$ depends on the sign of the difference between the propagation constants of the modes, which permits the coupling between each pair of them. If $\beta_{0 \mathrm{j}}>\beta_{0 \mathrm{k}}$, the minus sign is selected; otherwise the plus sign is chosen. The other two exponential functions in expression (12) consider the imaginary part of the leaky cladding modes.

The transmission can be found by assuming that only one mode is incident $\left(\mathrm{F}_{01}(0)=1\right.$ and $\left.\mathrm{F}_{02}(0)=\cdots \cdot \cdot=\mathrm{F}_{0 \mathrm{~N}}(0)=0\right)$ and solving the differential equation. The transmission power at the end of the LPFG can be expressed as:

$\frac{\left|F_{01}(L)\right|^{2}}{\left|F_{01}(0)\right|^{2}}$

where $\mathrm{L}$ is the length of the grating.

If only the self coupling coefficients and the cross coupling coefficients between the core mode and the cladding modes are considered, the computational effort is one order of magnitude lower, but additional errors can be caused for gratings with strong refractive index modulation [16]. For this reason, only the full matrix formulation is used. In addition to this, the selection of modes is critical in the reduction of computational effort and some rules are presented in [17].

\section{Methods for calculation of resonance displacement}

In the analysis of section III, one of the main purposes is to see the displacement of the resonance wavelengths as the parameter to detect experiments a variation. For this reason, alternative solutions to the application of coupled mode equations can be used if it is only necessary to analyze the displacement of the resonance wavelength. In this way computational effort is reduced.

The first one is the calculation of the resonance wavelength with the Bragg condition: 
$\beta_{01}(\lambda)-\beta_{0 j}(\lambda)=\frac{2 \pi}{\Lambda}$

where $\beta_{01}$ and $\beta_{0 \mathrm{j}}$ are the propagation constants of the core and the $\mathrm{j}$ cladding modes respectively, and $\Lambda$ is the period of the grating. Results obtained present appreciable variations related to those values calculated with coupled mode equations. However, if the modified first-order Bragg condition is applied, errors are lower than $0.1 \%$ [16]:

$\beta_{01}(\lambda)+s_{0} \zeta_{01,01}(\lambda)-\left(\beta_{0 j}(\lambda)+s_{0} \zeta_{0 j, 0 j}(\lambda)\right)=\frac{2 \pi}{\Lambda}$

If this error is compared with fabrication tolerances, it can be concluded that this approximation offers great advantages in terms of computational effort. Henceforward it will be used in some cases in next section.

If the overlay presents a complex refractive index, the self coupling coefficients will vary as light propagates in ' $\mathrm{z}$ ' direction. Consequently, the middle value of the self coupling coefficient between the beginning and the end of the grating was calculated:

$\varsigma_{0 j, 0 j}^{\prime}=\frac{1-\exp \left[-\operatorname{imag}\left(2 \beta_{0 j}\right) L\right\rfloor}{\operatorname{imag}\left(2 \beta_{0 j}\right) L} \varsigma_{0 j, 0 j}$

In order to apply expression (15) when the overlay presents a complex refractive index, $\varsigma_{0 j, 0 j}^{\prime}$ replaces $\varsigma_{0 j, 0 j}$.

\section{ANALYSIS OF LPFG STRUCTURES WITH DEPOSITED MATERIAL}

A commercial LPFG is selected for the analysis performed in this section. A modulation of the core refractive index is induced in a SMF28 single mode fiber. The parameters of the LPFG are: core diameter of $8.3 \mathrm{um}$, cladding diameter $125 \mathrm{um}$, core refractive index 1.47, cladding refractive index 1.4647, overlay refractive index $1.62+0.004 \mathrm{i}\left(\left[\mathrm{PDDA}^{+} / \mathrm{PolyR}^{-47}\right]\right)$, period of the grating is $276 \mu \mathrm{m}$, and the length of the grating is $25 \mathrm{~mm}$. The modulation is considered sinusoidal. Consequently $\sigma(z)=s_{0}=s_{1}=1$. The amplitude of the modulation is $2.85 \times 10^{-4}$. The notation used for the modes is: core mode $\mathrm{LP}_{01}$, first order cladding mode is $\mathrm{LP}_{02}$, second order cladding mode is $\mathrm{LP}_{03}$ and so forth.

If an overlay of higher refractive index than the cladding is deposited on this LPFG, cladding modes shift their effective index to higher values as the thickness of the overlay is increased. If a thickness value is exceeded, the overlay guides a mode. The important variation in comparison with overlays with low imaginary refractive index is that not always the lowest order cladding mode (highest effective index mode) is guided by the overlay. If the imaginary part of the refractive index in the overlay is high enough, the second, the third or a higher order cladding mode is guided by the overlay. This causes a reorganization of the effective index of the rest of modes as the thickness of the overlay is increased. Cladding modes of higher order than the one that is guided by the overlay shift their effective index value towards the original effective index before deposition of the immediate lower order cladding mode. On the other hand, cladding modes of lower order than the one that is guided by the overlay will shift their effective index value backwards to its original value before deposition. The final result is that the effective index distribution of modes before deposition is recovered.

In Fig. 2a the effective index of the first twelve cladding modes is represented as a function of the overlay thickness for a fixed wavelength of $1200 \mathrm{~nm}$. At $280 \mathrm{~nm}$, the $\mathrm{LP}_{0,3}$ mode is guided by the overlay. The effective index of the $\mathrm{LP}_{0,8}$ mode will be now that of the $\mathrm{LP}_{0,7}$, the effective index of the $\mathrm{LP}_{0,7}$ mode will be that of the $\mathrm{LP}_{0,6}$, and so forth. However, the $\mathrm{LP}_{0,2}$ mode shifts in a different manner. Its effective index increases originally. But after the $\mathrm{LP}_{0,3}$ mode is guided it refuses to continue and recovers its original value before deposition.

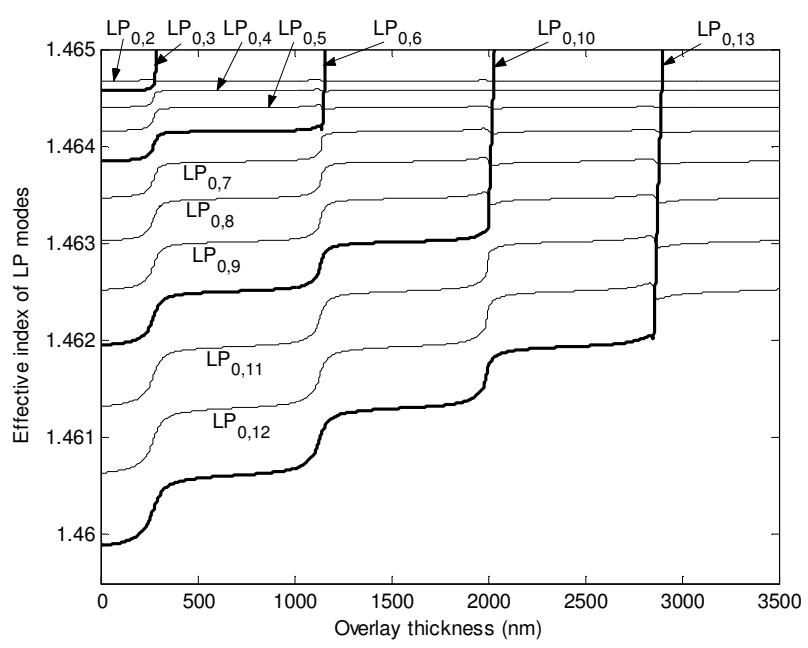

a)

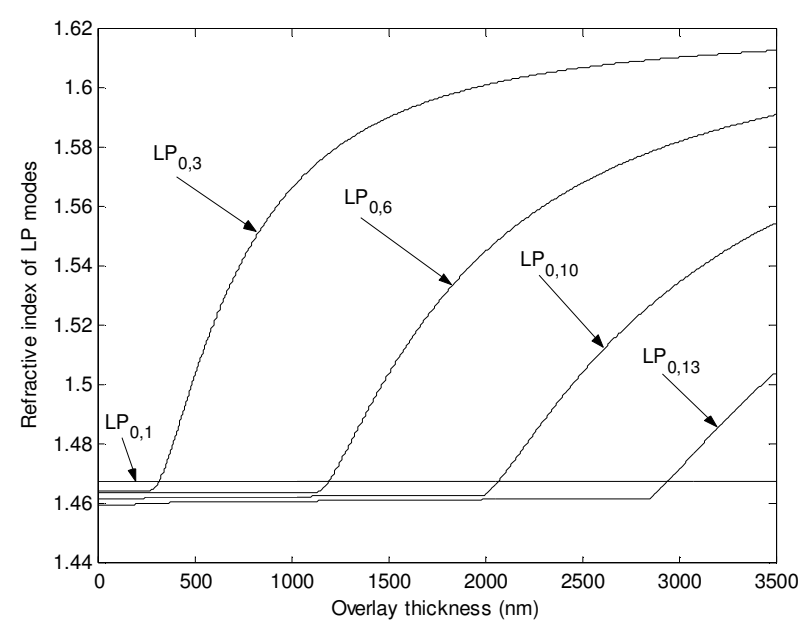

b)

Fig. 2. Effective index as a function of the overlay thickness of a) first twelve cladding modes and b) $\mathrm{LP}_{0,1}, \mathrm{LP}_{0,3}, \mathrm{LP}_{0,6}, \mathrm{LP}_{0,10}$ and $\mathrm{LP}_{0,13}$ modes.

The phenomenon is repeated each time a new mode is guided by the overlay, which implies new reorganizations of cladding modes. Moreover, the order of the cladding mode that is guided by the overlay is always higher or equal to the previous guided mode. And the same is true for the next guided modes. $\mathrm{LP}_{0,6}, \mathrm{LP}_{0,10}$ and $\mathrm{LP}_{0,13}$, become guided by the overlay at about 1120,1960 and $2800 \mathrm{~nm}$. The effective index 
of $\mathrm{LP}_{0,3} \mathrm{LP}_{0,6}, \mathrm{LP}_{0,10}$ and $\mathrm{LP}_{0,13}$ modes trends to the refractive index of the overlay (in this case 1.62) when each of them is guided by the overlay. To show this fact, these modes are represented with $\mathrm{LP}_{0,1}$ in Fig. $2 \mathrm{~b}$ in a wider scale than Fig. 2a.

The results obtained can be explained in terms of states of energy. There are allowed states for the effective indices of the modes. When the structure is perturbed by the deposition of an overlay, there exist not-allowed states that coincide with the transition to guidance of a cladding mode in the overlay. In lossless waveguides it is always the highest energy cladding mode (highest effective index), which is guided by the overlay.

The structure analyzed in this section is a lossy waveguide. As a result, the mode guided by the overlay may not be the highest state of energy. A lossy waveguide may guide a lower energy state. The thicker is the ESA region, the lossier becomes the waveguide. Consequently a lower effective index mode will be guided. Because of high losses there is no sense in obtaining the fields of cladding modes. However, fields were analyzed theoretically when no losses are present. The result is that higher order cladding modes than the one that is guided by the overlay mutate its field profile to that of next lower order cladding mode. The field profile of the sixth cladding mode becomes that of the fifth cladding mode, the fifth cladding mode profile changes to that of the fourth one, and so on. This explains the shift in the effective index of cladding modes in Fig. 2: there is a mutation of modes.

In addition to this, the imaginary effective index of the modes gives an important hint. In Fig. 3 the imaginary effective index of $\mathrm{LP}_{0,2}, \mathrm{LP}_{0,4}, \mathrm{LP}_{0,5} \mathrm{LP}_{0,7}, \mathrm{LP}_{0,8}$ is plotted. The common point of all modes is that there is a maximum in the imaginary part of all modes around the thickness values where cladding modes are guided by the overlay. This has important consequences in terms of vanishing of resonances as it will be explained later.

But there is another important idea that can be extracted from Fig. 3. Let us separate modes into two groups: lower and higher order cladding modes than the cladding mode that is guided by the overlay. The neighbor modes of the mode that becomes guided present the highest imaginary part in each of these two groups. This proves that the overlay attracts a specific state of energy and that the neighbor states also experience an influence of this attraction, which is appreciated in Fig. 3. At $280 \mathrm{~nm} \mathrm{LP}_{0,3}$ mode is guided. The group of lower order cladding modes is only $\mathrm{LP}_{0,2}$ mode, consequently its imaginary part is the maximum in this group. For the group of higher order cladding modes, $\mathrm{LP}_{0,4}$ shows the highest imaginary part. The same is true for the $\mathrm{LP}_{0,5}$ and $\mathrm{LP}_{0,7}$ modes at $1120 \mathrm{~nm}$, when $\mathrm{LP}_{0,6}$ is guided. At 1960 and $2800 \mathrm{~nm}$ the $\mathrm{LP}_{0,8}$ is the mode with higher imaginary part of those represented in Fig. 3, because it is closer to the modes with highest imaginary part at this two thickness values: $\mathrm{LP}_{0,9}$ and $\mathrm{LP}_{0,11}$ for $1960 \mathrm{~nm}$, and $\mathrm{LP}_{0,12}$ and $\mathrm{LP}_{0,14}$ for $2800 \mathrm{~nm}$.
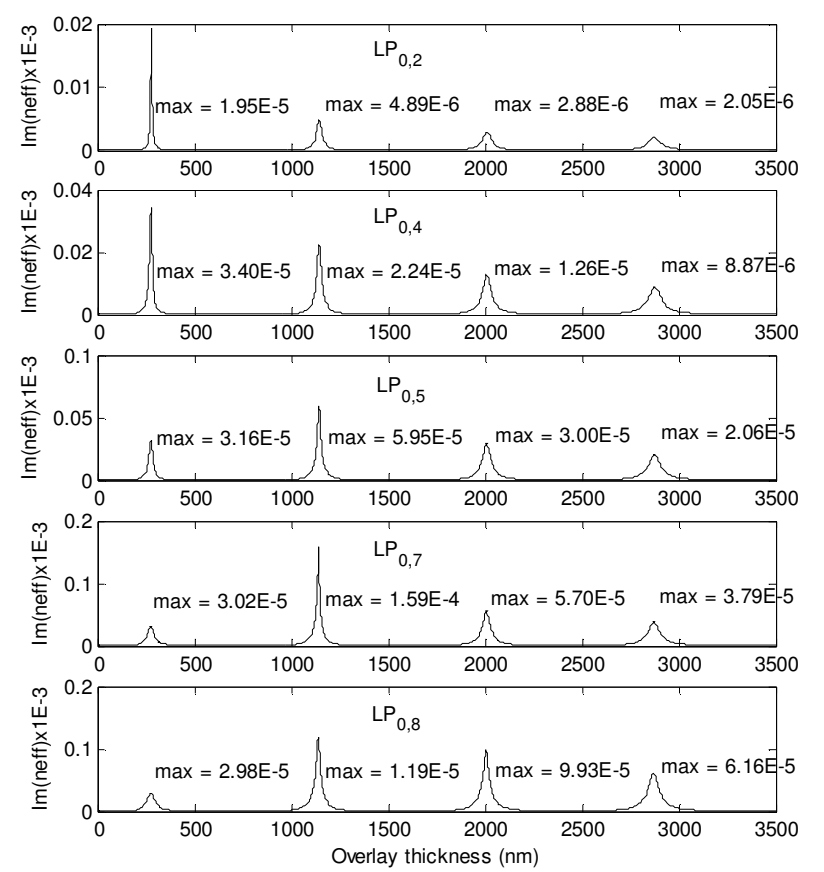

Fig. 3. Imaginary effective index as a function of the overlay thickness for $\mathrm{LP}_{0,2}, \mathrm{LP}_{0,4}, \mathrm{LP}_{0,5}, \mathrm{LP}_{0,7}$ and $\mathrm{LP}_{0,8}$.

Regarding the transmission spectrum of an LPFG, two important consequences are derived from the variation of the real and imaginary part of the cladding modes as an overlay is deposited on the cladding. The first one is that the shift in real effective index of the cladding modes leads to a displacement in all the attenuation bands. In analogy to the effective index, in Fig. 4 the attenuation band corresponding to the $\mathrm{LP}_{0,5}$ mode shifts its resonance wavelength to that of the $\mathrm{LP}_{0,4}$ mode and the same is true for the $\mathrm{LP}_{0,4}$ that shifts the wavelength to the attenuation band of the $\mathrm{LP}_{0,3}$. The same explanation is valid for the rest of attenuation bands corresponding to higher order cladding modes than the one that is guided by the overlay. This phenomenon was corroborated with experimental results in [13], and agrees with results of [12] and [24].

For lower order cladding mode resonances, there is a first shift to lower wavelengths. After a cladding mode is guided, the attenuation band recovers its original resonance wavelength; as it is the case in $\mathrm{LP}_{0,2}$ mode of Fig. 4 . In this figure, both coupled mode equations and modified Bragg condition are contrasted to show that the error introduced by the last one is very low.

$\mathrm{LP}_{0,3}$ resonance is not represented in Fig.4, because when the $\mathrm{LP}_{0,3}$ mode is guided by the overlay there is no longer a coupling with the core mode. Consequently, values obtained with the modified Bragg condition are only valid before the guidance at the overlay starts. 


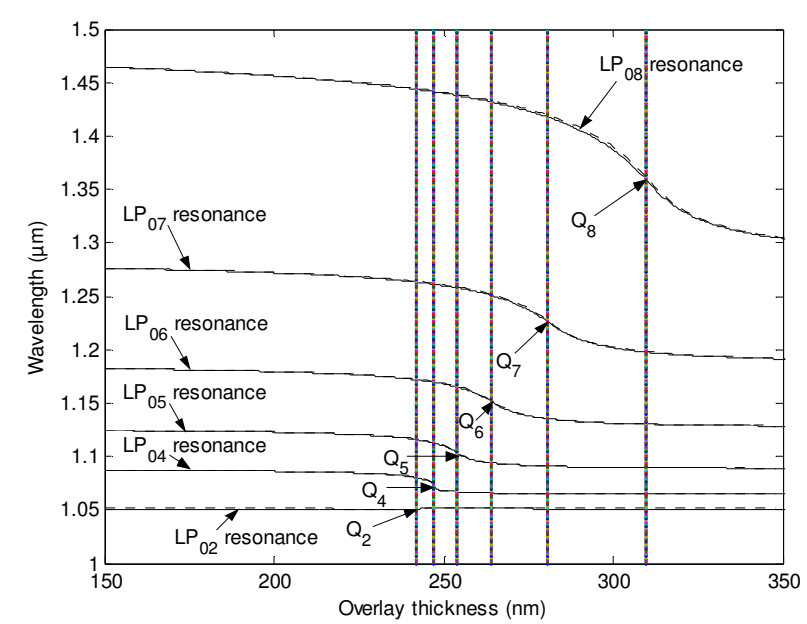

Fig. 4. $\mathrm{LP}_{0,2} \mathrm{LP}_{0,4} \mathrm{LP}_{0,5}, \mathrm{LP}_{0,6} \mathrm{LP}_{0,7}$ and $\mathrm{LP}_{0,8}$ resonances as a function of the thickness of the overlay. Overlay refractive index: 1.62+0.004i. Ambient index 1. Dotted lines: modified Bragg condition. Straight lines coupled mode equations

In addition to this, there is an optimal deposition thickness where the central wavelength shift as a function of the thickness of the overlay is highest. Henceforward the shift as a function of the ambient refractive index or the overlay refractive index is also highest. This is the optimum overlay thickness (OOT). This value depends mainly on two variables: the overlay and the ambient refractive indices. Consequently, a good choice for a specific ambient refractive index is to stop the deposition when the effective index value of a mode is located between the effective index of the mode itself before deposition, and that of the next lower cladding mode before deposition. This is an approximate solution. To calculate a more exact value, either the modified Bragg condition or the couple mode equations explained in section 2 will be used. In Fig. 4 the OOT for $\mathrm{LP}_{02}, \mathrm{LP}_{04}, \mathrm{LP}_{05}, \mathrm{LP}_{06}, \mathrm{LP}_{07}$ and $\mathrm{LP}_{08}$ is respectively $\mathrm{Q}_{2}=242, \mathrm{Q}_{4}=247, \mathrm{Q}_{5}=254, \mathrm{Q}_{6}=264, \mathrm{Q}_{7}=$ 280.5 and $\mathrm{Q}_{8}=309.5$. These values correspond with the maxima of the derivative of the wavelength of the resonance as a function of the overlay thickness.

The second consequence of the variation of the effective index of cladding modes is related to the imaginary part. In Fig. 3 it is proved that for all modes there is a maximum in the imaginary part of the effective index at the transition to guidance of one of the cladding modes in the overlay. The reason is that the field profile of the mode changes. This phenomenon is more important in higher order cladding modes than the one that is guided by the overlay, because the mode profile changes to the immediate lower cladding mode. At the middle of the mutation of the mode, the absolute value of the field is maximum at the interface between the cladding and the overlay. Since the overlay presents a complex refractive index, the cladding mode is more influenced when the field is maximum at the interface. In this way, a maximum in the imaginary part of the cladding mode is reached. When cladding modes present a negligible imaginary part, the coupling between the core mode and a cladding mode is constant along the grating. Consequently, at the output of the LPFG a part of the core mode power is transferred to the cladding mode. However, when cladding modes show a high imaginary part, there is a progressive reduction of the coupling between the cladding modes and the core mode along the LPG, because there is an attenuation factor in coupled mode equations (12). As a result, the core mode does not lose power at the output of the LPFG and a vanishing of the attenuation bands is caused. In Fig. 5, the minimum transmission of the attenuation bands is plotted as a function of the overlay thickness for $\mathrm{LP}_{02}, \mathrm{LP}_{04}, \mathrm{LP}_{05}$, and $\mathrm{LP}_{06}$ mode resonances. $\mathrm{LP}_{0,2}$ vanishes and recovers its original minimum transmission, $\mathrm{LP}_{0,4}$ vanishes and reaches the minimum transmission of $\mathrm{LP}_{0,3}$ mode before deposition started, $\mathrm{LP}_{0,5}$ vanishes and reaches the minimum transmission of $\mathrm{LP}_{0,4}$ resonance before deposition started and so on.

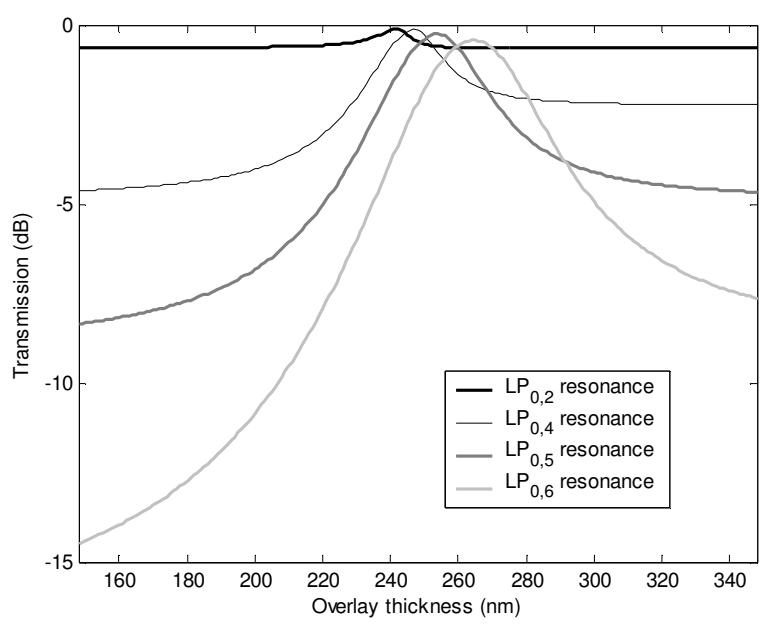

Fig. 5. Minimum transmission of $\mathrm{LP}_{0,2} \mathrm{LP}_{0,4} \mathrm{LP}_{0,5}$ and $\mathrm{LP}_{0,6}$ attenuation bands as a function of the thickness of the overlay. Overlay refractive index: $1.62+0.004$ i.. Ambient index 1 .

The vanishing of modes has been experimentally corroborated [14]. In Fig. 6 experimental results of the minimum transmission of $\mathrm{LP}_{0,7}$ and $\mathrm{LP}_{0,8}$ attenuation bands are contrasted with theoretical ones. The phenomenon of vanishing is clearly appreciated. The depth of the attenuation band is not the same mainly because of the LP approximation, which in higher order cladding modes is not so exact, and because a sinusoidal grating is assumed in theory, which is not exactly the case after UV irradiation. However, the wavelength of completely vanishing is well reproduced in both modes analyzed.

After seeing this property of deposition of materials with high imaginary part, the OOT is limited to those thickness values where the attenuation band is still appreciable. Otherwise our detection system loses the signal. Anyway, even out of the OOT, shifts in resonance wavelength improve in a great manner the sensitivity of LPFGs towards the ambient or overlay refractive index. Furthermore, spectra can be analyzed in terms of depth of the attenuation band, which varies in an important way. 

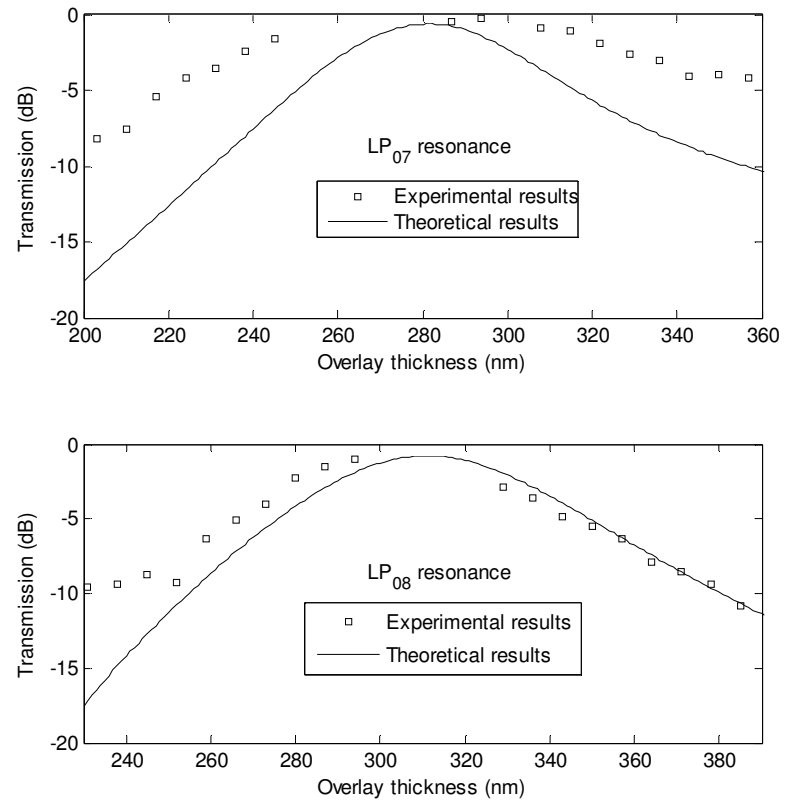

Fig. 6. Minimum transmission of $\mathrm{LP}_{0,7}$ and $\mathrm{LP}_{0,8}$ attenuation bands as a function of the overlay thickness. Theoretical and experimental results.

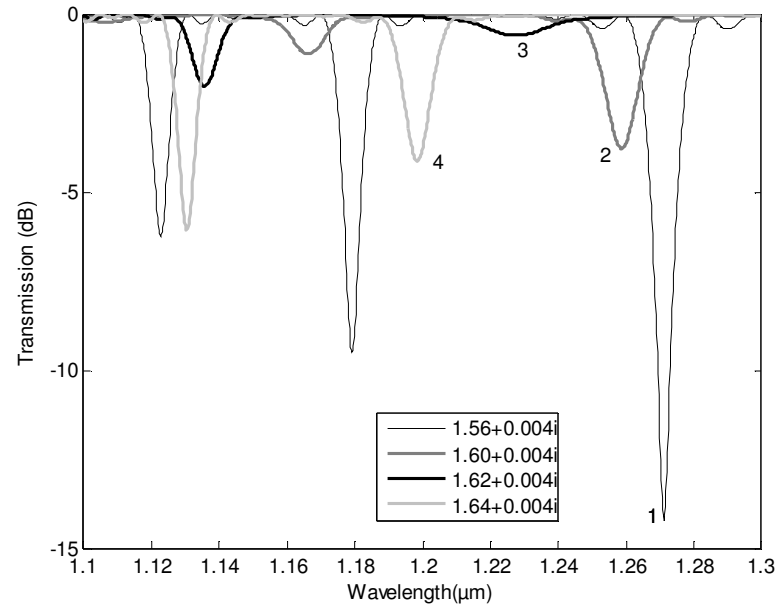

Fig. 7. Transmittance spectra of $\mathrm{LP}_{0,5}, \mathrm{LP}_{0,6}$ and $\mathrm{LP}_{0,7}$ mode resonances of an LPFG as a function of four overlay refractive indices: $1.56+0.004 \mathrm{i}$, $1.6+0.004 \mathrm{i}, 1.62+0.004 \mathrm{i}$ and $1.64+0.004 \mathrm{i}$. Ambient refractive index: 1 .

Finally, in Fig. 7 the shift in wavelength and the variation in the minimum transmission of the attenuation bands of $\mathrm{LP}_{0,5}$, $\mathrm{LP}_{0,6}$ and $\mathrm{LP}_{07}$ modes is represented. In this case it is proved that the phenomenon is also dependent on the refractive index of the overlay, and not only on its thickness. To this purpose, the transmission spectra of an LPFG are analyzed for four refractive index values of the overlay: $1.56+0.004 \mathrm{i}$, $1.6+0.004 \mathrm{i}, 1.62+0.004 \mathrm{i}$ and $1.64+0.004 \mathrm{i}$. The ambient refractive index is 1 . The thickness of the overlay is $280.5 \mathrm{~nm}$. This value is the OOT of the $\mathrm{LP}_{0,7}$ resonance for a refractive index of the overlay of $1.62+0.004 \mathrm{i}$. At $1.56+0.004 \mathrm{i}$, resonance wavelength and depth are similar to that before deposition (point 1 of Fig. 7). At $1.60+0.004 \mathrm{i} \mathrm{LP}_{0,7}$ mode shifts towards the original position of $\mathrm{LP}_{0,6}$ mode (point 2 of Fig. 7). At $1.62+0.004 \mathrm{i}$ the attenuation band vanishes (point 3 of Fig. 7), which coincides with OOT for this resonance, and it reappears at $1.64+0.004 \mathrm{i}$ close to the $\mathrm{LP}_{0,6}$ mode attenuation band without deposition (point 4 of Fig. 7). A similar effect can be appreciated in $\mathrm{LP}_{0,6}$ resonance.

Apart from the imaginary part of the overlay material, other additional effects may contribute to the vanishing of attenuation bands, such as radiation losses of the cladding modes. In this work it has been proved that the imaginary part of the refractive index of the material deposited is very important and permits to predict experimental results.

\section{CONCLUSION}

Calculation of the LP modes in a multilayer waveguide and coupled mode equations permits to obtain the transmission spectrum in an LPFG with an overlay. The approximation is valid for low order LP modes.

It has been demonstrated that if the overlay presents a higher refractive index than the core, it guides modes if a thickness value is exceeded. In the transition to guidance of each of these modes there is a fast shift of the resonance wavelength of the attenuation bands obtained in the transmission spectrum. Those attenuation bands corresponding to higher order cladding modes than the one that is guided by the overlay shift to the immediate lower order cladding mode resonance. For lower order cladding mode resonances than the one that is guided, the attenuation band firstly shifts to lower wavelengths and after the cladding mode is guided it recovers its original resonance wavelength before deposition. This phenomenon has been interpreted in terms of energy states.

The sensitivity of the LPFG to ambient or overlay refractive index can be increased in a great manner if an appropriate thickness of the overlay is selected. However, both in Electrostatic Self-Assembly (ESA) Monolayer process and Langmuir Blodgett (LB) nanodeposition techniques there is an additional phenomenon of vanishing of the attenuation bands at a specific range of overlay thickness values. This limits the thickness values of the overlay if the design is aimed for a detection of wavelength shift. But it adds the possibility of detecting changes in the minimum transmission of the attenuation band instead of changes in wavelength. By considering a complex refractive index for the overlay material experimental results are reproduced theoretically. Other factors may also contribute to this phenomenon such as radiation losses of cladding modes, but have not been considered in this work.

In our opinion, deposition of overlays on the cladding of LPFGs will permit to extend even more the applications of these structures. Furthermore the theory presented in this work could be also applied to other structures where nanodeposition is applied.

\section{ACKNOWLEDGMENT}

This work was supported by Spanish Ministerio de Ciencia y Tecnologia and FEDER Research Grants CICYT-TIC 200300909, Gobierno de Navarra and FPU MECD Grant. We 
thank Philippe Lalanne of the Institut d'Optique Centre National de la Recherche Scientifique, France for his useful comments, encouragement, and work.

\section{REFERENCES}

[1] J. R.Qiang and H. E. Chen, "Gain flattening fibre filters using phase shifted long period fibre grating," Electron. Lett., vol. 34, pp. 1132$1133,1998$.

[2] A. M. Vengsarkar, P. J. Lemaire, J. B. Judkins, V. Bathia, T. Erdogan, and J. E. Sipe, "Long-period fiber gratings as Band Rejection Filters," $J$. Lightwave Technol., vol. 14, pp. 58-65, 1996.

[3] B. J. Eggleton, R. E. Slusher, J. B. Judkins, J. B. Stark and A. M. Vengsarkar, "All-optical switching in long period fiber gratings," Opt. Lett., vol. 22, pp. 883-885, 1997.

[4] K. W. Chung, S. Yin, "Analysis of widely tunable long-period grating by use of an ultrathin cladding layer and higher-order cladding mode coupling," Opt. Lett., vol. 29, pp. 812-814, 2004.

[5] V. Bathia and A. M. Vengsarkar, "Optical fiber long-period grating sensors," Opt. Lett., 21, pp. 692-694, 1996.

[6] V. Bhatia, "Applications of long-period gratings to single and multi parameter sensing," Opt. Exp. vol. 4, pp. 457-466, 1999.

[7] Y. G. Han, S. B. Lee, C. S. Kim, J. U. Kang, U. C. Paek and Y. Chung, "Simultaneous measurement of temperature and strain using dual longperiod fiber gratings with controlled temperature and strain sensitivities," Opt. Exp., vol. 11, pp. 476-481 2003.

[8] C. C. Ye, S. W. James and R. P. Tatam, "Simultaneous temperature and bend sensing using using long-period fiber gratings," Opt. Lett., vol. 25, pp. 1007-1009, 2000.

[9] H. J. Patrick, A. D. Kersey and F. Bucholtz, "Analysis of the response of long period fiber gratings to external index of refraction," J. Lightwave Technol., vol. 16, pp. 1606-1612, 1998.

[10] R. Hou, Z. Ghassemlooy, A. Hassan, C. Lu and K. P. Dowker, "Modelling of long-period fibre grating response to refractive index higher than that of cladding," Meas. Sci. Technol., vol. 12, pp. 17091713, 2001.

[11] S. T. Lee, R. D. Kumar, P. S. Kumar, P. Radhakrishnan, C. P. G. Vallabhan, V. P. N. Nampoori, "Long period gratings in multimode optical fibers: application in chemical sensing," Opt. Comm., vol. 224, pp. 237-241, 2003.

[12] N. D. Rees, S. W. James, R. P. Tatam and G. J. Ashwell, "Optical fiber long-period gratings with Langmuir-Blodgett thin-film overlays," Opt. Lett., vol. 27, pp. 686-688, 2002.

[13] M. Achaerandio, F. J. Arregui and I. R. Matías, "Electrostatic selfassembled thin films deposited on optical fiber long-period gratings for the fabrication of chemical sensors," Proc. of the SPIE, vol. 5502, pp. 300-303, 2004.

[14] I. Del Villar, M. Achaerandio, I. R. Matias and F. J. Arregui, "Deposition of an Overlay with Electrostactic Self-Assembly Method in Long Period Fiber Gratings," Opt Lett., In press.

[15] S. W. James and R. P. Tatam, "Optical fibre long-period grating sensors: characteristics and application," Meas. Sci Technol. vol. 14, pp. R49-R61, 2003.

[16] E. Anemogiannis, E. N. Glytsis and T. K. Gaylord, "Transmission characteristics of long- period fiber gratings having arbitrary azimutal/radial refractive index variation," J. Lightwave Technol., vol. 21, pp. 218-227, 2003.

[17] T. Erdogan, "Cladding-mode resonances in short- and long-period fiber gratings filters," J. Opt. Soc. Am. A, vol. 14, pp. 1760-1773, 1997.

[18] D. B. Stegall and T. Erdogan, "Leaky cladding mode propagation in long-period fiber grating devices," IEEE Photon. Technol. Lett., vol. 11, pp. 343-345, 1999.

[19] Y. Koymada, "Numerical analysis of core-mode to radiation-mode coupling in long-period fiber gratings," IEEE Photon. Technol. Lett. vol. 13, pp. 308-310, 2001.
[20] I. Del Villar, I. R. Matias, F. J. Arregui and R. O. Claus, "ESA based infiber nanocavity for hydrogen peroxide detection," IEEE Trans. Nanotech., In press.

[21] G. Stewart and B. Culshaw, "Optical waveguide modelling design for evanescent field chemical sensor," Opt. Quant. Elec., vol. 26, pp. S249S259, 1994.

[22] D. Gloge, "Weakly guiding fibers," App. Opt., vol. 10, pp. 2252-2258, 1971.

[23] K. Morishita, "Numerical analysis of pulse broadening in grated index optical fibers," IEEE Trans. Microwave Theory Tech., vol. MTT-29, pp. 348-352, 1981.

[24] A. W. Snyder and J. D. Love, "Optical waveguide theory," London U.K: Chapman and Hall, 1983.

Ignacio Del Villar received his MS degree in Electrical and Electronic Engineering in 2002 from the Public University of Navarra (UPNA) and is pursuing the Ph.D. in the Electrical and Electronic Engineering Department of the Public University of Navarra.

During 2004 he was a visiting scientist at the Institut d'Optique Centre National de la Recherche Scientifique BP 147 Orsay 91403 France.

His research interests include analysis and fabrication of optical fiber sensors and nanostructures. He has served as a referee for the journals Optical Express, JOSA A, IEEE Sensors Journal and Sensors \& Actuators B.

Ignacio R. Matías received his MS degree in Electrical and Electronic Engineering and his Ph.D. degree, speciality in Optical Fiber Sensors in 1992 and 1996, respectively from the Polytechnic University of Madrid (UPM), Spain.

In 1996 he took up a lectureship at the Public University of Navarra (Pamplona, Spain) where presently he is a Permanent Professor.

He has co-authored more than one hundred journal and conference papers related to optical fiber sensors, passive optical devices and systems. He is an IEEE member. He is an Associate Editor of the IEEE Sensors Journal.

Francisco J. Arregui (M'01) received the M.S. degree in electrical engineering from the Catholic University of Navarra (San Sebastian, Spain) in 1994 and the Ph.D. degree from the Public University of Navarra (Pamplona, Spain) in 2000.

He was a member of the CEIT Research Center (San Sebastian, Spain) for two years and since 1995 has been working at the Public University of Navarra where currently is an Associate Professor. During 1998, 2000 and 2004 he has also been a visiting scientist at the Fiber \& Electro Optics Research Center, Virginia Tech, (Blacksburg, VA, USA).

Dr. Arregui has served as a referee for several journals including Optical Engineering, Sensors \& Actuators A, Sensors \& Actuators B, IEEE Photonics Technology Letters, IEEE Journal of Lightwave Technology and IEEE Sensors Journal. His main research interests include optical fiber sensors, sensor materials and nanostructured materials. Francisco J. Arregui is a member of IEEE and SPIE.

Miguel Achaerandio received his MS degree in Physics, speciality in Optics, in 1993 from the University of Zaragoza, and is pursuing both the MS degree in Electrical and Electronic Engineering and the Ph.D. in the Public University of Navarra.

His research interests include the fabrication of optical fiber sensors and nanostructures. 